\title{
Catalytic depolymerisation of suberin rich biomass with precious metal catalysts
}

DOI:

10.1039/C8GC00605A

\section{Document Version}

Final published version

Link to publication record in Manchester Research Explorer

\section{Citation for published version (APA):}

McCallum, C. S., Strachan, N., Bennett, S. C., Graham Forsythe, W., Garrett, M. D., Hardacre, C., Morgan, K., \& Sheldrake, G. N. (2018). Catalytic depolymerisation of suberin rich biomass with precious metal catalysts. Green Chemistry, 20(12), 2702-2705. https://doi.org/10.1039/C8GC00605A

\section{Published in:}

Green Chemistry

\section{Citing this paper}

Please note that where the full-text provided on Manchester Research Explorer is the Author Accepted Manuscript or Proof version this may differ from the final Published version. If citing, it is advised that you check and use the publisher's definitive version.

\section{General rights}

Copyright and moral rights for the publications made accessible in the Research Explorer are retained by the authors and/or other copyright owners and it is a condition of accessing publications that users recognise and abide by the legal requirements associated with these rights.

\section{Takedown policy}

If you believe that this document breaches copyright please refer to the University of Manchester's Takedown Procedures [http://man.ac.uk/04Y6Bo] or contact uml.scholarlycommunications@manchester.ac.uk providing relevant details, so we can investigate your claim.

\section{OPEN ACCESS}




\title{
PARP inhibitors in platinum-sensitive high-grade serous ovarian cancer
}

\author{
Robert D. Morgan ${ }^{1,2} \cdot$ Andrew R. Clamp ${ }^{1,2} \cdot$ D. Gareth R. Evans ${ }^{3} \cdot$ Richard J. Edmondson $^{4,5} \cdot$ Gordon C. Jayson $^{1,2}$
}

Received: 23 November 2017 / Accepted: 25 January 2018

(C) The Author(s) 2018. This article is an open access publication

\begin{abstract}
Purpose Poly(ADP-ribose) polymerase inhibitors (PARPi) have changed the management of high-grade serous ovarian cancer (HGSOC). The rationale for the development of PARPi was based on the concept of synthetic lethality, in which a cell can survive a deficiency of one gene/gene product, but may die if there is a deficiency in a combination of genes/gene products. In women with $B R C A 1 / 2$ deficiency within their ovarian cancer tissue, inhibition of PARP imposes an intolerable burden of DNA damage repair deficiency and may induce cell death.

Methods Clinical trials have evaluated PARPi as single-agent therapeutics and as maintenance treatment following platinumbased chemotherapy for HGSOC. Clinical data suggest the most impressive anti-tumour activity occurs in women with platinum-sensitive ovarian cancer and germline or somatic BRCAl/2 mutations (g/sBRCAmt).

Results In the maintenance setting, randomised trials have shown that PARPi compared to placebo reduce the hazard ratio for the development of progressive disease to $0.2-0.27$ for patients with a $\mathrm{g} / \mathrm{s} B R C A \mathrm{mt}$; to $0.34-0.38$ for patients with putative evidence of DNA damage repair deficiency; and to $0.35-0.45$ in an unselected population with HGSOC. Furthermore, phase $1 / 2$ trials have reported single-agent anti-tumour response rates in $\mathrm{g} B R C A \mathrm{mt}$ of approximately $50 \%$ in platinum-sensitive and $25 \%$ in platinum-resistant disease.

Conclusion Here, we discuss the evidence for the use of PARPi as single-agent therapeutics and maintenance treatment in HGSOC and evaluate the genetic assays used in clinical trials so far. We discuss the emerging role of platinum sensitivity as a broad eligibility criteria for the use of PARPi.
\end{abstract}

Keywords Ovarian cancer · Platinum sensitivity · PARP inhibitors · BRCA mutation

Gordon C. Jayson

gordon.jayson2@christie.nhs.uk

1 Department of Medical Oncology, The Christie NHS Foundation Trust, Manchester, UK

2 Manchester Cancer Research Centre, The University of Manchester, Wilmslow Road, Manchester M20 4BX, UK

3 Division of Evolution and Genomic Sciences, Manchester Academic Health Science Centre, University of Manchester, Manchester, UK

4 Department of Obstetrics and Gynaecology, St Mary's Hospital, Central Manchester NHS Foundation Trust, Manchester, UK

5 Division of Cancer Sciences, Faculty of Biology, Medicine and Health, University of Manchester, Manchester, UK

\section{Introduction}

Epithelial ovarian cancer is the fourth commonest cause of female cancer-related death in the west [1]. The commonest histological subtype, high-grade serous carcinoma, accounts for approximately $70 \%$ of cases [1]. Epithelial ovarian cancer often presents with advanced disease. Primary treatment is tailored to individual patients but often involves cytoreductive surgery followed by platinum-based chemotherapy. In a minority of patients with advanced stage disease, this approach is associated with long-term survival, but sadly the majority will develop recurrent disease within 12-18 months of their primary treatment.

Upon disease relapse, treatment switches to controlling symptoms and improving survival with sequential lines of platinum- and/or taxane-based chemotherapy, often resulting in diminishing disease-free intervals until drug resistance develops and palliation is required. The 5-year survival for 
ovarian cancer is approximately $35 \%$ [2]. Two drug classes of maintenance therapy have so far been developed [3]. The first group is the VEGF inhibitors, which largely improve progression-free survival (PFS). Improvements in overall survival (OS) have only been reported in occasional trials or trial subgroups [4].

The second class of maintenance therapy agents are the poly(ADP-ribose) polymerase inhibitors (PARPi) [5]. These small molecule inhibitors exploit the susceptibility of cancer cells to defects in DNA damage repair and have shown impressive results in clinical trials as both single-agent therapeutics and maintenance treatments. Herein, we discuss the use of PARPi in high-grade serous ovarian carcinoma and the genetic assays that have been assessed as potential predictive biomarkers.

\section{Homologous recombination repair}

Following the initial description of the sensitivity of $B R C A$ -deficient cells to PARPi [6, 7], further work tested the hypothesis that impairments in double-strand DNA repair might predict for sensitivity to PARPi. Homologous recombination (HR) repair is a high-fidelity genetic recombination process involved in the repair of DNA double-strand breaks (DSBs) [8]. It occurs during the G2/S phase of the cell cycle where the presence of a sister chromatid/homologous chromosome allows error-free repair of DSBs and preservation of genomic integrity [8]. Germline loss-of-function (LOF) monoallelic mutations in genes involved in HR repair such as BRCA1, BRCA2, RAD51C, RAD51D and BRIPI are associated with an increased risk of developing familial ovarian cancer [9-15]. For carriers of the high-penetrance cancersusceptibility genes $B R C A 1$ and $B R C A 2$, the life-time risk of ovarian cancer, and particularly high-grade serous carcinoma, is between $40-70$ and $20-50 \%$, respectively $[16,17]$. Malignant transformation in BRCA-related familial ovarian cancer is thought to occur when the remaining wild-type allele undergoes pathogenic mutation or epigenetic silencing, thereby leaving only the germline $B R C A 1 / 2$ mutant allele [18].

A deficiency in $B R C A 1$ or $B R C A 2$ within a cell is believed to impair HR repair, termed homologous recombination deficiency (HRD), and place a greater reliance upon alternative DNA repair pathways to repair DSBs, such as non-homologous end joining (NHEJ) and microhomology-mediated end joining (MMEJ) [19]. These alternative pathways are of low fidelity and error-prone, with potential for losses or gains of nucleotide bases within the cell's genome during the repair process [20]. A reliance on NHEJ and/or MMEJ in cells with $B R C A 1 / 2$ deficiency increases the probability of mutations occurring within the genome, a process known as genomic instability, and may ultimately lead to malignant transformation [21]. The BRCA1/2 genes are, therefore, considered tumour suppressor genes and are likely to require biallelic LOF for tumorigenesis to occur; in keeping with Knudson's double-hit hypothesis [22-24]. In women with a somatic BRCA1/2 mutation in the tumour tissue only, biallelic LOF occurs through mutations and/or epigenetic silencing in both wild-type alleles and is often clonal [25, 26]. The relationship between possessing a germline monoallelic mutation in other non-BRCA HR repair genes and genomic instability/ tumorigenesis is less clear.

\section{PARP inhibitors}

PARP are a family of cellular enzymes that are involved in a variety of biological functions. The most abundant and well-characterised members are PARP-1 and PARP-2, which have a role in DNA damage detection and repair [27]. Within their catalytic site, nicotinamide adenine dinucleotide is used as a substrate to form polymers of ADP-ribose in a process called poly (ADP-ribosyl)ation or PARylation [28]. PARP binds to DNA at single-strand breaks (SSBs) and forms PAR on itself and other accessory proteins associated with DNA. These polymers are then able to recruit proteins involved in the base excision repair (BER) pathway; utilised to repair SSBs [29].

Small molecule inhibitors of PARP-1/2 were engineered to inhibit the catalytic domain at nanomolar concentrations. By inhibiting PARP, SSBs remain unrepaired and may potentially form lethal DSBs as the ensuing replication fork stalls and/or collapses [19]. More recent evidence also suggests that PARPi trap PARP on DNA and thereby prevent the dissociation required for the BER pathway to proceed [30-32]. There is also evidence to suggest that PARP is involved in NHEJ and that dysregulation of NHEJ may determine PARPi sensitivity [33, 34].

Preclinical work has convincingly demonstrated that cells deficient in BRCA1/2 were sensitive to PARP inhibition. The proposed mechanism for this effect is synthetic lethality, in which a cell is able to survive with a deficiency in one gene/ gene product but may die if a deficiency occurs in a particular combination of two or more genes/gene products [35, 36]. A deficiency in BRCAl/2 is believed to impair a cell's ability to repair DSBs through HR repair. A lethal second deficiency is brought about by targeted pharmacological inhibition of PARP-1/2 [19].

The use of PARPi is changing how high-grade serous ovarian carcinoma is treated. PARP inhibitors have been evaluated in two distinct clinical settings; as single-agent therapeutics and as maintenance treatment following a response to platinum-based chemotherapy. 


\section{Single-agent therapy}

PARPi single-agent therapy has been thoroughly investigated in phase $1 / 2$ trials in women with ovarian cancer (see Table 1) [37-53]. These trials predominantly enrolled women with high-grade serous ovarian carcinoma and a germline BRCA1/2 mutation (gBRCAmt). Most women had been pre-treated with several lines of traditional cytotoxic chemotherapy and in most trials platinum sensitivity was not mandated. A range of doses of individual PARPi were used in the search for recommended phase $2 / 3$ doses. Nevertheless, data from these trials reported encouraging anti-tumour responses across all PARPi in selected groups

Table 1 Objective response rates (ORR) as per RECIST (CR/PR) to PARPi single-agent therapy in ovarian cancer

\begin{tabular}{|c|c|c|c|c|}
\hline Clinical trial & PARPi & Dose & $B R C A$ status & ORR, \% (95\% CI) \\
\hline \multicolumn{5}{|l|}{ Phase 1 trials } \\
\hline Fong et al. $^{\mathrm{a}}$ & Olaparib & $200 \mathrm{mg} \mathrm{BD}^{\mathrm{d}}$ & $\mathrm{g} B R C A \mathrm{mt}$ & $\begin{array}{l}\text { Plat-Sen } 46 \\
\text { Plat-Res } 33\end{array}$ \\
\hline Mateo et al. ${ }^{\mathrm{a}}$ & Olaparib & $\begin{array}{l}300 \mathrm{mg} \mathrm{BD}^{\mathrm{e}} \\
400 \mathrm{mg} \mathrm{BD}^{\mathrm{e}}\end{array}$ & $\mathrm{g} B R C A \mathrm{mt}$ & $\begin{array}{l}\text { Plat-Sen \& Plat-Res } 38^{\mathrm{g}} \\
\text { Plat-Sen \& Plat-Res } 42^{\mathrm{g}}\end{array}$ \\
\hline Kristeleit et al. & Rucaparib & $40 \mathrm{mg}$ OD $-840 \mathrm{mg} \mathrm{BD}$ & $\mathrm{g} B R C A \mathrm{mt}$ & $\begin{array}{l}\text { Plat-Sen } 12 \\
\text { Plat-Res } 9\end{array}$ \\
\hline Sandhu et al. ${ }^{\mathrm{a}}$ & Niraparib & $300 \mathrm{mg}$ OD & $\begin{array}{l}\text { BRCAmt } \\
\text { Sporadic HGSOC }\end{array}$ & $\begin{array}{l}\text { Plat-Sen } 50 \\
\text { Plat-Res } 33 \\
\text { Plat-Sen } 33 \\
\text { Plat-Res } 5\end{array}$ \\
\hline De Bono et al. ${ }^{\mathrm{a}}$ & Talazoparib & $0.025-1.1 \mathrm{mg} /$ day OD & $\mathrm{g} B R C A \mathrm{mt}$ & $\begin{array}{l}\text { Plat-Sen } 55 \\
\text { Plat-Res } 20\end{array}$ \\
\hline \multicolumn{5}{|l|}{ Phase 2 trials } \\
\hline Auden et al. & Olaparib & $400 \mathrm{mg}$ BD & $\mathrm{g} B R C A \mathrm{mt}$ & $\begin{array}{l}\text { Plat-Sen } 38 \\
\text { Plat-Res } 30\end{array}$ \\
\hline Gelmon et al. & Olaparib & $400 \mathrm{mg} \mathrm{BD}$ & $\begin{array}{l}\text { BRCAmt } \\
\text { BRCAwt or unknown } \\
\text { HGSOC }\end{array}$ & $\begin{array}{l}\text { Plat-Sen } 60 \\
\text { Plat-Res } 33 \\
\text { Plat-Sen } 50 \\
\text { Plat-Res } 4\end{array}$ \\
\hline Kaye et al. ${ }^{\mathrm{b}}$ & Olaparib & $400 \mathrm{mg} \mathrm{BD}$ & $\mathrm{g} B R C A \mathrm{mt}$ & Plat-Sen \& Plat-Res $31^{\mathrm{g}}$ \\
\hline Kaufman et al. & Olaparib & $400 \mathrm{mg} \mathrm{BD}$ & $\mathrm{g} B R C A \mathrm{mt}$ & Plat-Res only $31(25-38)$ \\
\hline Drew et al. & Rucaparib & Variable oral dosing levels ${ }^{\mathrm{f}}$ & $\mathrm{g} B R C A \mathrm{mt}$ & Plat-Sen \& Plat-Res $21^{\mathrm{g}}$ \\
\hline Kristeleit et al. & Rucaparib & $600 \mathrm{mg}$ BD & $B R C A \mathrm{mt}$ & Plat-Sen 60 (43-74) \\
\hline ARIEL2 (Part 1) & Rucaparib & $600 \mathrm{mg} \mathrm{BD}$ & $\begin{array}{l}\text { BRCAmt } \\
B R C A w \mathrm{w} / \mathrm{LOH}^{\text {High }} \\
B R C A w \mathrm{w} / \mathrm{LOH}^{\mathrm{Low}}\end{array}$ & $\begin{array}{l}\text { Plat-Sen } 80(64-91) \\
\text { Plat-Sen } 29(20-40) \\
\text { Plat-Sen } 10(4-20)\end{array}$ \\
\hline Coleman et al. & Veliparib & $400 \mathrm{mg} \mathrm{BD}$ & $\mathrm{g} B R C A \mathrm{mt}$ & 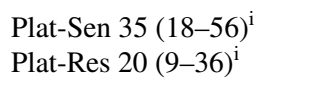 \\
\hline Steffensen et al. ${ }^{\mathrm{c}}$ & Veliparib & $300 \mathrm{mg} \mathrm{BD}$ & $\mathrm{g} B R C A \mathrm{mt}$ & $\begin{array}{l}\text { Plat-Sen } 56 \\
\text { Plat-Res } 45\end{array}$ \\
\hline
\end{tabular}

$B R C A m t$ pathogenic $B R C A 1 / 2$ mutation, $B R C A w t$ wild type $B R C A 1 / 2, B D$ twice daily, $C R$ complete response, $H G S O C$ high-grade serous ovarian cancer, $L O H$ loss of heterozygosity, $n$ number of patients, $O D$ once daily, $P R$ partial response, Plat-Sen platinum sensitivity, Plat-Res platinum resistant

${ }^{\text {a } P h a s e ~} 1$ dose expansion study

${ }^{\mathrm{b}}$ Randomised phase 2 trial

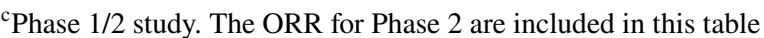

${ }^{\mathrm{d}}$ The majority of patients $(n=39 / 50)$ received $200 \mathrm{mg}$ twice daily. Dose levels ranged from $40 \mathrm{mg}$ once daily for 2 out of every 3 weeks to $600 \mathrm{mg}$ twice daily

${ }^{\mathrm{e}}$ ORR for the tablet formulation (150 mg tablet), as opposed to capsule formulation, is reported in this table

${ }^{\mathrm{f}}$ Only patients receiving oral rucaparib included in this table; oral doses ranged from $92 \mathrm{mg}$ once daily for 7 days q21 days to $600 \mathrm{mg}$ twice daily continuous daily dosing

${ }^{\mathrm{g}}$ Combined ORR for patients with platinum-sensitive or platinum-resistant disease

${ }^{\mathrm{h}}$ Study included women with platinum-resistant disease and those not suitable for further platinum therapy

${ }^{\mathrm{i}} 90 \%$ confidence interval 
of patients. Patients with $\mathrm{g} B R C A \mathrm{mt}$ often had better antitumour responses than those with wild-type $B R C A 1 / 2$ (BRCAwt) and patients with platinum-sensitive disease had better responses than women with platinum-resistant or refractory disease. Data also suggested that women with platinum-resistant disease who carried a gBRCAmt achieved better objective response rates (ORRs) than those with $B R C A$ wt.

The most impressive anti-tumour response reported was in the phase 2, single arm, multi-centre trial, ARIEL2 (Part 1) in which women with a germline or somatic $B R C A$ $\mathrm{mt}(\mathrm{g} / \mathrm{s} B R C A \mathrm{mt})$ achieved a RECIST ORR [complete/partial response (CR/PR)] of $80 \%$ (95\% CI 62-97\%) with rucaparib single-agent therapy [53]. The vast majority of women recruited had high-grade serous ovarian cancer and all women had a progression-free interval following the penultimate platinum-based chemotherapy of at least 6 months. The median number of prior lines of chemotherapy was 2 (inter quartile range [IQR] of 1 to 2). In the same trial, women with $B R C A w t$ had a more modest, but encouraging, RECIST ORR (CR/PR) of $21 \%$ (the objective response by combined RECIST and CA-125 was $35 \%$ ).

Across all phase 1/2 trials investigating PARPi as a single-agent therapy women with platinum-sensitive ovarian cancer achieved better ORRs compared to those with platinum-resistant/refractory disease (see Table 1). In addition, a number of trials demonstrated that a longer platinum-free interval (PFI) correlated with a higher likelihood of achieving an anti-tumour response [41, 43, 47, 54].

Data from a number of phase $1 / 2$ trials also suggested that in the platinum-resistant setting, eligibility for PARPi singleagent therapy might appropriately include $B R C A 1 / 2$ mutational status. Indeed, the ORRs for women with a $\mathrm{g} B R C A$ $\mathrm{mt}$ and platinum-resistant disease were noticeably higher (approximately 20-30\%) across all PARPi than that seen in women with BRCAwt (see Table 1). In a phase-1 dose expansion trial assessing niraparib, Sandhu et al. reported an ORR of 5\% in women with platinum-resistant sporadic high-grade serous ovarian or primary peritoneal cancer compared to $33 \%$ in women who had a $\mathrm{g} B R C A \mathrm{mt}$ [51]. In addition, in a non-randomised phase 2 trial assessing olaparib, Gelmon et al. reported an ORR as per RECIST (CR/PR) of $33 \%$ in women with a $\mathrm{g} B R C A \mathrm{mt}$ and platinum-resistant high-grade serous or undifferentiated ovarian carcinoma, but a much lower ORR of $4 \%$ in those with BRCAwt [44]. Interestingly, in the same trial Gelmon et al. also showed that all women with a $\mathrm{g} B R C A \mathrm{mt}$ and platinum-resistant disease achieved a CA-125 treatment response compared to only $40 \%$ with $B R C A$ wt. These data may suggest that the acquisition of platinum-resistance in women with $B R C A \mathrm{wt}$ is associated with PARPi single-agent therapy resistance, but that the presence of a $\mathrm{g} B R C A \mathrm{mt}$ maintains PARPi sensitivity. Women with $B R C A$ wt and platinum-resistant disease may, therefore, benefit more from combinational therapy with PARPi and additional targeted treatments, anti-angiogenic inhibitors and/or immune therapy [55-57].

As single agents, PARPi brought about modest and manageable toxicities. Specific grade 3-4 adverse events (AEs) included most commonly anaemia but also fatigue, nausea and vomiting. These AEs are now recognised class effects of which the majority are grade 1-2. Rucaparib caused a noticeable comparable increase in grade 3-4 ALT/AST ( $>10 \%)$ although these increases often occurred within the first few weeks after starting treatment and were not associated with any symptoms, infrequently led to treatment discontinuation $(<1 \%)$, and, reversed following dose omission [58]. The recommended phase $2 / 3$ dose of niraparib (300 mg once daily) did cause notable myelosuppression [51]. Rare $(\leq 1 \%)$ but important class-specific AEs also included myelodysplastic syndrome/acute myeloid leukaemia (MDS/ AML) and pneumonitis. The incidence of MDS/AML was similar across all PARPi and did not depend upon duration of treatment [37, 45, 51, 54].

A number of trials are ongoing to assess PARPi as singleagent therapy in the platinum-sensitive and platinum-resistant setting (see Table 2).

\section{Maintenance therapy}

More recently, the utilisation of PARPi has focused on maintenance treatment following a response to platinum-based chemotherapy. Several phase 2/3 trials have assessed PARPi as maintenance treatment in women with relapsed highgrade ovarian cancer [59-63] (see Table 3). In each trial, PARPi were commenced following a treatment response [RECIST (CR/PR) \pm CA-125 response] to platinum therapy. These trials primary outcome was progression-free survival (PFS); either investigator assessed or blinded independent central review (BICR; see Table 3). In addition, secondary efficacy outcomes included time to first subsequent therapy (TFST) and time to second subsequent therapy (TSST) as there was concern that platinum-resistance may be induced by PARPi and so these exploratory endpoints allow analysis of this (see Table 4).

\section{Randomised phase 2 maintenance therapy trial}

Study 19, a randomised, placebo-controlled, phase 2 trial, first assessed PARPi as maintenance treatment in ovarian cancer (see Table 3) [60]. All enrolled women had recurrent, platinum sensitive (defined as a progression-free interval of 6 months or more from the penultimate platinum chemotherapy) high-grade serous ovarian carcinoma and had achieved a CR/PR to their latest platinum-based chemotherapy. Patients were randomised to receive either 
Table 2 Ongoing phase 2/3 trials assessing PARPi as single-agent therapy and maintenance monotherapy

Single-agent therapy

SOLO-3 (NCT02282020)

OCTOVA (NCT03117933)

QUADRA (NCT02354586)

ARIEL2 Part 2 (NCT01891344)

ARIEL4 (NCT02855944)
Olaparib vs. chemotherapy (gBRCAmt, platinum-sensitive)

Olaparib \pm cediranib vs. chemotherapy (g/s BRCAmt, platinum-resistance)

Niraparib after 3 or 4 lines of chemotherapy (single arm, phase 2)

Rucaparib after $\geq 3$ lines of chemotherapy (single arm, phase 2)

Rucaparib vs. chemotherapy (g/s BRCAmt, platinum-sensitive or resistance)

\begin{tabular}{ll}
\hline SOLO-1 (NCT01844986) & $\begin{array}{c}\text { Olaparib vs. placebo (g/sBRCAmt, following response to first-line platinum therapy) } \\
\text { OReO (NCT03106987) }\end{array}$ \\
& $\begin{array}{c}\text { to repeat platinum therapy) } \\
\text { Niraparib vs. placebo (following response to first-line platinum-based therapy) } \\
\text { Veliparib vs. placebo (following concurrent first-line carboplatin, paclitaxel \& veli- } \\
\text { parib) }\end{array}$
\end{tabular}

$g$ germline, $g / s$ germline or somatic

Table 3 Progression-free survival (PFS) in phase 2/3 trials assessing PARPi as maintenance treatment

\begin{tabular}{|c|c|c|c|c|c|}
\hline Clinical Trial & Subgroups & Study arms & PFS/months (95\% CI) & HR $(95 \% \mathrm{CI})$ & Pvalue \\
\hline \multirow[t]{2}{*}{ Study $19^{a}$} & HGSOC & Olaparib vs. Placebo & 8.4 vs. 4.8 & $0.35(0.25-0.49)$ & $<0.001$ \\
\hline & $\mathrm{g} / \mathrm{s} B R C A \mathrm{mt}$ & Olaparib vs. Placebo & $11.2(8.3$-NC) vs. $4.3(3.0-5.4)$ & $0.18(0.10-0.31)$ & $<0.0001$ \\
\hline $\mathrm{SOLO}_{2}{ }^{\mathrm{a}}$ & $\mathrm{g} B R C A \mathrm{mt}$ & Olaparib vs. Placebo & $19.1(16.3-25.7)$ vs. $5.5(5.2-5.8)$ & $0.30(0.22-0.41)$ & $<0.0001$ \\
\hline \multirow[t]{3}{*}{ NOVA $^{\mathrm{b}}$} & $\mathrm{g} B R C A \mathrm{mt}$ & Niraparib vs. Placebo & 21.0 vs. 5.5 & $0.27(0.17-0.41)$ & $<0.001$ \\
\hline & $\begin{array}{l}\text { Non-gBRCAmt / } \\
\text { HRD-carcinoma }\end{array}$ & Niraparib vs. Placebo & 12.9 vs. 3.8 & $0.38(0.24-0.59)$ & $<0.001$ \\
\hline & Non-gBRCAmt & Niraparib vs. Placebo & 9.3 vs. 3.9 & $0.45(0.34-0.61)$ & $<0.001$ \\
\hline \multirow[t]{3}{*}{ ARIEL $^{\mathrm{a}}$} & $\mathrm{g} / \mathrm{s} B R C A \mathrm{mt}$ & Rucaparib vs. Placebo & $16.6(13.4-22.9)$ vs. $5.4(3.4-6.7)$ & $0.23(0.16-0.34)$ & $<0.0001$ \\
\hline & HRD carcinoma & Rucaparib vs. Placebo & $13.6(10.9-16.2)$ vs. $5.4(5.1-5.6)$ & $0.32(0.24-0.42)$ & $<0.0001$ \\
\hline & ITT population & Rucaparib vs. Placebo & $10.8(8.3-11.4)$ vs. $5.4(5.3-5.5)$ & $0.36(0.30-0.45)$ & $<0.0001$ \\
\hline
\end{tabular}

$C I$ confidence interval, $g / s B R C A m t$ germline or somatic, $H G S O C$ high-grade serous ovarian carcinoma, HRD Homologous Recombination Deficiency, ITT intention to treat, $N C$ not calculable

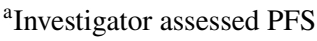

${ }^{\mathrm{b}}$ Blinded independent central review PFS

olaparib $400 \mathrm{mg}$ twice daily (50 $\mathrm{mg}$ capsules) or placebo. The median number of previous chemotherapy regimens in the experimental arm was 3 (range 0-11). Investigators reported that olaparib led to a significantly longer PFS than placebo (8.4 vs. 4.8 months, HR $0.35,95 \%$ CI $0.25-0.49$, $P<0.001$ ) [60]. A subsequent pre-planned retrospective analysis showed that median PFS was even longer in patients with $\mathrm{g} / \mathrm{s} B R C A \mathrm{mt}$ (11.2 vs. 4.3 months, HR $0.18,95 \% \mathrm{CI}$ 0.10-0.31, P <0.0001) (see Table 3) [61]. Although the study showed a significant improvement in overall survival (OS) in the primary analysis (HR $0.73,95 \%$ CI $0.55-0.96$, $P=0.025$ ) particularly in women with $\mathrm{g} / \mathrm{s} B R C A \mathrm{mt}$ (HR 0.62 , $95 \%$ CI $0.41-0.94, P=0.025)$, these results are considered descriptive as the trial was not originally powered to detect a difference in OS [64, 65].

\section{Phase 3 maintenance treatment trials}

Three randomised, placebo-controlled, double-blinded phase 3 trials have investigated PARPi as maintenance treatment in women with ovarian cancer (see Table 3) [59, 62, 63]. All patients recruited had recurrent, platinum sensitive (defined as a progression-free interval of 6 months or more from the penultimate platinum chemotherapy) high-grade serous or endometrioid ovarian cancer and had achieved a CR/PR to their last platinum-based chemotherapy. Approximately $60 \%$ of patients had received two previous lines of chemotherapy before study enrolment and $60 \%$ had a PFI of at least 12 months. All participants were enrolled within 8 weeks of their last cycle of platinum. 
Table 4 Median time to first subsequent therapy (TFST) and time to second subsequent therapy (TSST) reported in phase 2/3 trials assessing PARPi as maintenance treatment

\begin{tabular}{|c|c|c|c|c|}
\hline Clinical trial & Subgroups & Study arms & TFST/months (95\% CI) & TSST/months (95\% CI) \\
\hline \multirow[t]{2}{*}{ Study 19} & HGSOC & Olaparib vs. Placebo & $\begin{array}{l}13.4(11.3-15.7) \text { vs. } 6.7(5.7-8.2) \\
\text { HR } 0.39(0.29-0.51) \\
P<0.0001\end{array}$ & $\begin{array}{l}19.1(16.5-22.0) \text { vs. } 14.8(14.0-17.2) \\
\text { HR } 0.52(0.39-0.68) \\
P<0.0001\end{array}$ \\
\hline & $\mathrm{g} / \mathrm{s} B R C A \mathrm{mt}$ & Olaparib vs. Placebo & $\begin{array}{l}15.6(12.3-28.2) \text { vs. } 6.2(5.3-9.2) \\
\text { HR } 0.32(0.22-0.48) \\
P<0.0001\end{array}$ & $\begin{array}{l}22.0(17.7-34.9) \text { vs. } 15.3(14.0-18.7) \\
\text { HR } 0.41(0.28-0.62) \\
P<0.0001\end{array}$ \\
\hline SOLO2 & $\mathrm{g} B R C A \mathrm{mt}$ & Olaparib vs. Placebo & $\begin{array}{l}27.9(22.6-\mathrm{NC}) \text { vs. } 7.1(6.3-8.3) \\
\text { HR } 0.28(0.21-0.38) \\
P<0.0001\end{array}$ & $\begin{array}{l}\text { NR }(\text { NC) vs. } 18.2(15.0-20.5) \\
\text { HR } 0.37(0.26-0.53) \\
P<0.0001\end{array}$ \\
\hline \multirow[t]{2}{*}{ NOVA } & $\mathrm{g} B R C A \mathrm{mt}$ & Niraparib vs. Placebo & $\begin{array}{l}21(17.5-\mathrm{NR}) \text { vs. } 8.4(6.6-10.6) \\
\text { HR } 0.31(0.21-0.48) \\
P<0.001\end{array}$ & Not reported \\
\hline & Non-gBRCAmt & Niraparib vs. Placebo & $\begin{array}{l}11.8(9.7-13.1) \text { vs. } 7.2(5.7-8.5) \\
\text { HR } 0.55(0.41-0.72) \\
P<0.001\end{array}$ & Not reported \\
\hline
\end{tabular}

$C I$ confidence interval, $H R$ Hazard Ratio, $N R$ not reached, $N C$ not calculable

In SOLO2/ENGOT-Ov21 (see Table 3: olaparib vs. placebo), only women with a $\mathrm{g} / \mathrm{s} B R C A \mathrm{mt}$ were eligible to participate, although no women with a somatic $B R C A$ mutation were enrolled [63]. The dose and formulation of olaparib assessed in SOLO2 (300 mg twice daily in 100 or $150 \mathrm{mg}$ tablets) differed from Study 19 (400 mg twice daily in $50 \mathrm{mg}$ capsules) following phase $1 / 2$ data showing improved bioavailability with $300 \mathrm{mg}$ twice daily in a tablet formulation compared to $400 \mathrm{mg}$ twice daily in a capsule formulation [49]. In ENGOT-OV16/NOVA [62] (see Table 3: niraparib vs. placebo), eligible patients had less than $2 \mathrm{~cm}$ of residual disease and normalisation of CA-125 following the last cycle of platinum therapy. In ARIEL3 [59] (rucaparib vs. placebo) no restrictions were made regarding the volume of residual disease following the last cycle of platinum chemotherapy and women with any persistent lesion greater than $2 \mathrm{~cm}$ diameter were defined as having bulky disease (rucaparib arm 19\%; placebo 15\%).

All three trials reported a significant improvement in the primary outcome of median PFS in women receiving PARPi compared to placebo (see Table 3). NOVA reported the longest PFS in the experimental arm (21.0 months; BICR PFS).
In addition, in SOLO2 and ARIEL3 additional anti-tumour responses were reported beyond those achieved with the latest platinum therapy, confirming the cytotoxic effect of PARPi demonstrated in single-agent therapeutic trials (see Table 5) [58, 59].

The most encouraging results from NOVA and ARIEL3 were that cohorts of women with or without a $\mathrm{g} / \mathrm{s} B R C A$ mt achieved a significantly improved PFS compared with patients who received placebo. Niraparib and rucaparib improved the median PFS by 5.4 months in the non-germline BRCA1/2 mutation group (NOVA) and the intention-to-treat (ITT) population (ARIEL3) demonstrating the efficacy of the drugs in a genetically unselected population (see Table 3 ).

The data from these trials, in combination with the primary analysis from Study 19, demonstrate that PARPi are likely to benefit women with recurrent, platinum-sensitive high-grade serous ovarian cancer with or without a $\mathrm{g} / \mathrm{s} B R C A$ mt. As all participants in these trials had platinum-sensitive disease this may be an appropriate and broader predictive biomarker to screen eligibility for PARPi.

There were some initial concerns that PARPi maintenance therapy may lead to platinum-resistance [66-70].
Table 5 Objective response rates (ORR) as per RECIST criteria (CR/PR) for PARPi as maintenance treatment

\begin{tabular}{llll}
\hline Clinical Trial & PARP inhibitor & Subgroups & ORR, \% $(n)$ \\
\hline Study 19 & Olaparib 400 mg BD vs. placebo & HGSOC & 12 (7/57) vs. 4 (2/48) \\
SOLO2 & Olaparib 300 mg BD vs. placebo & gBRCAmt & 41 (30/73) vs. 17 (6/35) \\
ARIEL3 & Rucaparib 600 mg BD vs. placebo & g/s BRCAmt & $38(15 / 40)$ vs. 9 (2/23) \\
& & HRD carcinoma & 27 (23/85) vs. 7 (3/41) \\
& & ITT population & $18(26 / 141)$ vs. 8 (5/66) \\
\hline
\end{tabular}

$B D$ twice daily, $g$ germline, $g / s$ germline or somatic, HRD Homologous Recombination Deficiency, ITT intention-to-treat 
In those phase 3 trials with mature data, the median TFST and TSST were significantly longer in women treated with PARPi compared to placebo (see Table 4) [59, 61, 63, 71]. The TFST in women with a $\mathrm{g} B R C A \mathrm{mt}$ was consistently in excess of 12 months across trials. Furthermore, the significant improvement in TSST across trials provides a surrogate marker of improved OS before full maturation of data. Thus, overall, there are no significant concerns that PARPi treatment induces platinum-resistance in a clinical setting.

As maintenance therapy, PARPi class-based AEs of fatigue, nausea, vomiting and anaemia were again evident. There was noticeably higher grade 3-4 neutropenia, thrombocytopenia and hypertension in patients receiving niraparib and grade 3-4 increases in ALT/AST in patients receiving rucaparib (see Table 6). The incidence of MDS/ AML was around $1 \%$ across all PARPi. Safety data were not reported for individual cohorts of women and so it is unclear if the incidence and severity of off-target AEs was worse in women with a $\mathrm{g} B R C A \mathrm{mt}$ vs. sBRCAmt or $B R C A$ wt. Adverse events appeared to be manageable with dose reductions/interruptions and did not significantly affect quality of life [72-75].
Ongoing trials are also assessing the use of PARPi maintenance treatment as part of first-line chemotherapy as well as re-challenge PARPi maintenance treatment (see Table 2) [76-78].

\section{Genetic biomarkers}

The clinical development of PARPi was predicted on the need for functional PARP1/2 in the absence of BRCA1/2 function or other causes of homologous recombination deficiency (HRD). In keeping with these observations, genetic assays were investigated in NOVA and ARIEL3 to predict response to PARPi. Up to $50 \%$ of women with high-grade serous ovarian carcinoma may harbour a mutation in, or epigenetic silencing of, genes involved in HR repair [14, 79-84] and preclinical data suggested cells deficient in BRCA1/2 and non-BRCA HR repair genes are sensitive to PARPi [6, $7,10,85,86]$. Other surrogate markers of HRD proposed as predictive biomarkers for PARPi include genetic mutations within the cancer genome suggestive of a greater reliance on alternative error-prone DNA damage repair mechanisms
Table 6 Key treatment-related adverse events in phase 3 trials assessing PARPi as maintenance treatment

\begin{tabular}{|c|c|c|c|c|c|c|}
\hline & \multicolumn{2}{|c|}{$\begin{array}{l}\text { SOLO2 } \\
\text { Olaparib } 300 \text { mg BD }\end{array}$} & \multicolumn{2}{|c|}{$\begin{array}{l}\text { NOVA } \\
\text { Niraparib } 300 \mathrm{mg} \text { OD }\end{array}$} & \multicolumn{2}{|c|}{$\begin{array}{l}\text { ARIEL3 } \\
\text { Rucaparib } 600 \mathrm{mg} \\
\text { BD }\end{array}$} \\
\hline & $\mathrm{G} 1-2(\%)$ & $\mathrm{G} 3-4(\%)$ & Any grade $(\%)$ & $\mathrm{G} 3-4(\%)$ & $\mathrm{G} 1-2(\%)$ & G3-4 (\%) \\
\hline \multicolumn{7}{|c|}{ Haematological adverse events } \\
\hline Anaemia & 24 & 19 & 50 & 25 & 19 & 19 \\
\hline Neutropenia & 14 & 5 & 30 & 20 & 11 & 7 \\
\hline Thrombocytopenia & 13 & 1 & 61 & 34 & 13 & 5 \\
\hline \multicolumn{7}{|c|}{ Gastrointestinal adverse events } \\
\hline Nausea & 73 & 3 & 74 & 3 & 72 & 4 \\
\hline Vomiting & 35 & 3 & 34 & 2 & 33 & 4 \\
\hline Diarrhoea & 32 & 1 & 19 & $<1$ & 31 & 1 \\
\hline Abdominal pain & 22 & 3 & 23 & 1 & 27 & 2 \\
\hline Constipation & 21 & 0 & 40 & $<1$ & 35 & 2 \\
\hline Dyspepsia & 11 & 0 & 11 & 0 & 14 & $<1$ \\
\hline \multicolumn{7}{|l|}{ Other key adverse events } \\
\hline Fatigue & 62 & 4 & 59 & 8 & 63 & 7 \\
\hline Dysgeusia & 27 & 0 & 10 & 0 & 39 & 0 \\
\hline Headache & 25 & 1 & 25 & $<1$ & 18 & $<1$ \\
\hline Decreased appetite & 22 & 0 & 25 & $<1$ & 23 & 1 \\
\hline Dyspnea & 11 & 1 & 19 & 1 & 13 & 0 \\
\hline Insomnia & 6 & 0 & 24 & $<1$ & 14 & 0 \\
\hline Hypertension & 3 & 0 & 19 & 8 & NR & NR \\
\hline Increase ALT/AST & 5 & 3 & 10 & 4 & 23 & 10 \\
\hline Increase blood creatinine & 11 & 1 & $\mathrm{NR}^{\mathrm{a}}$ & $\mathrm{NR}^{\mathrm{a}}$ & 15 & $<1$ \\
\hline
\end{tabular}

$A L T$ alanine aminotransaminase, $A S T$ aspartate aminotransaminase, $B D$ twice daily, $N R$ not reported, $O D$ once daily

${ }^{a}$ FDA label for Niraparib states increase in blood creatinine identified in $\geq 1 \%$ to $<10 \%$ participants but grades not reported 
such as NHEJ and/or MMEJ $[19,87]$. These putative biomarkers include genome-wide loss of heterozygosity $(\mathrm{LOH})$, telomeric allelic imbalance (TAI) and large-scale state transitions (LST). Loss of heterozygosity is the irreversible loss of one parental allele [88]. Telomeric allele imbalances are regions of allelic imbalance that extend to one of the subtelomeres but do not cross the centromere [89]. Large-scale state transitions are chromosomal breaks between two adjacent regions of at least $10 \mathrm{Mb}$ after filtering out regions less than $3 \mathrm{Mb}[90]$. Both BRCA1/2 deficiency $[89,91]$ and sensitivity to platinum therapy [90] have been associated with greater $\mathrm{LOH}$ and increased regions of TAI and LST.

Next generation sequencing (NGS) assays can detect mutations in non-BRCA HR repair genes as well as the presence of LOH, TAI and/or LST in DNA extracted from tumour tissue. Two of these assays were prospectively evaluated in NOVA and ARIEL3, including Myriad myChoice ${ }^{\circledR}$ HRD test [62] (Myriad Genetics, Inc., UT, US) and Foundation Medicine T5 NGS assay [59] (Foundation Medicine, Inc., MA, US) respectively. The comparative results of these two technologies are presented below. These NGS-based assays may determine the presence/absence of mutations in genes involved in HR repair but do not provide information on function $[92,93]$.

\section{Myriad myChoice ${ }^{\circledR}$ HRD test}

Myriad's myChoice ${ }^{\circledR}$ HRD test was assessed in NOVA as a predictive biomarker for niraparib maintenance treatment [62]. In women without a $\mathrm{g} B R C A \mathrm{mt}$ (non-gBRCAmt), DNA was extracted from archival ovarian tumour tissue and sequenced for somatic mutations in BRCA1/2 and 54,091 genome-wide single nucleotide polymorphisms (SNPs) to quantify LOH, TAI and LST [62]. In this assay, genomic LOH was specifically defined as the number of $\mathrm{LOH}$ regions greater than $15 \mathrm{Mb}$ within the tumour genome, but less than a whole chromosome [91]. The assay calculates an algorithmic score (range 0 to 100) based on the accumulation of $\mathrm{LOH}$, TAI and LST and produces a HRD "status". All patients with a sBRCAmt and/or an algorithmic score of 42 or above were classified as HRD-positive, suggestive of HR deficiency [94, 95]. Patients with non-gBRCAmt and HRD-positive were enrolled into an independent cohort for the primary outcome analysis of BICR PFS (see Table 3). In addition, investigators assessed the primary outcome in all women with non-g $B R C A$ mt including those with sBRCAmt, HRD-positive and HRDnegative tumour tissue (see Table 3 ).

\section{Foundation Medicine T5 NGS assay}

The Foundation Medicine T5 NGS assay was assessed in ARIEL3 as a predictive biomarker for rucaparib maintenance treatment [59]. This assay was used to detect mutations in $30 \mathrm{HR}$ repair genes and to quantify genomewide $\mathrm{LOH}$ (as a percentage) by sequencing over 3500 evenly distributed SNPs in DNA extracted from archival ovarian tumour tissue [96]. The assay was unable to differentiate between $\mathrm{g} / \mathrm{s} B R C A \mathrm{mt}$ so blood could also be tested for germline mutations using the BRACAnalysis CDx test (Myriad Genetics, Inc., UT, US). Any patient found to have a g/ $\mathrm{s} B R C A \mathrm{mt}$ or genomic $\mathrm{LOH}$ of $16 \%$ or more were grouped together in a predefined cohort called "HRD carcinoma" for assessment of the primary outcome of investigator-assessed PFS (see Table 3). The cut off of $16 \%$ for LOH was determined following a retrospective analysis of ARIEL2 (Part 1) in which a clinically significant benefit in PFS was achieved in patients treated with rucaparib single-agent therapy who had at least $16 \%$ genomic $\mathrm{LOH}[53,97]$. The trial investigators in ARIEL3 also assessed the primary outcome in the ITT population, including all patients with either a $\mathrm{g} / \mathrm{s} B R C A$ mt or $B R C A$ wild-type $/ \mathrm{LOH}^{\mathrm{High}}$ or $B R C A$ wild-type $/ \mathrm{LOH}^{\mathrm{Low}}$ (see Table 3).

Neither the Myriad myChoice ${ }^{\circledR}$ HRD test nor Foundation Medicine T5 NGS assay could fully predict which patients with relapsed, platinum-sensitive high-grade serous ovarian cancer were likely to benefit from niraparib or rucaparib maintenance therapy, respectively (see Table 3 ). The results from these trials raise important questions about the utility of genetic assays as predictive biomarkers in this selected group of patients. Both trials showed a tiered treatment effect, with the greatest benefit in PFS achieved in women with a gBRCAmt, however they also demonstrated that most women with platinum-sensitive high-grade serous ovarian carcinoma benefit from maintenance PARPi. This suggests that a conceptually simpler and much less expensive predictive biomarker for PARPi therapy could be platinum sensitivity.

The clinical relevance of possessing a germline or somatic mutation in a non-BRCA gene involved in HR repair as a predictive biomarker for PARPi also remains unclear. Data from both ARIEL2 (Part 1) and ARIEL3 reported around $10 \%$ of patients with high-grade serous ovarian cancer had a germline or somatic mutation in a non-BRCA HR repair gene $[53,59]$. This represents a potential sizeable target population. Nevertheless, between these trials only patients with germline/somatic RAD51C or RAD51D mutations consistently achieved treatment responses to rucaparib [53, 98]. Indeed, the presence of a mutation in $R A D 51 C$ or $R A D 51 D$ correlated with an ORR (as per RECIST [CR/PR] and/or CA-125) to single-agent therapy with rucaparib in ARIEL2 (Part 1) and also led to encouraging median PFS [rucaparib: 16.4 months (range 5.4-30.4 months) versus placebo: 5.4 months (range 3.9-5.5 months)] with rucaparib maintenance treatment $[53,98]$. Interestingly, all $R A D 51 C / D$ mutations recorded in ARIEL2 (Part 1) and ARIEL3 correlated with the putative marker of genomic scarring " $\mathrm{LOH}^{\mathrm{High}}$ ", 
and, in ARIEL3 were reported as homozygous, suggesting biallelic LOF may bring about genomic instability and predispose to PARPi sensitivity. As a notable comparison approximately $80-90 \%$ of $\mathrm{g} B R C A \mathrm{mt}$ carriers with ovarian cancer are reported to have $\mathrm{LOH}$ of the wild-type allele; in keeping with biallelic LOF and consistent with Knudson's double hit hypothesis for tumour suppressor genes [23, $80,82]$. Therefore, if NGS-based assays are to be used on tumour tissue to determine eligibility for PARP inhibitors, it may be appropriate to consider screening for biallelic LOF (i.e. homozygosity) in RAD51C and RAD51D as well as $B R C A 1$ and $B R C A 2$.

\section{Circulating tumour DNA as genetic biomarkers}

Despite women with platinum-sensitive high-grade serous ovarian cancer achieving a significant PFS benefit to PARPi, the majority eventually develop progressive disease. A number of mechanisms of resistance to PARPi have been reported including intragenic reversions in the germline mutant alleles, loss of 53BP1, reduction in PAR expression and up-regulation of P-glycoprotein efflux pumps (reviewed in Lord et al. [99]). In clinical practice, the most frequently reported resistance mechanism is secondary reversion mutations/intragenic deletions in germline $B R C A l[100,101]$ or $B R C A 2[67-69,100]$ mutations following platinum-based therapy or in germline BRCA1 [102], BRCA2 [66], RAD51C and $R A D 51 D$ [102] mutations following PARPi therapy. The incidence of $B R C A 1 / 2$ reversion mutations in women with platinum-resistant ovarian cancer has been reported as high as $46 \%$ (95\% CI 29-65\%) [100]. In women with sBRCA1/2 mutations, copy number alternation and up-regulation of the remaining wild-type allele has also been suggested as potential resistance mechanisms to PARPi [103]. Multiple reversions in germline mutations have been described in the same patient at different sites of metastatic disease as well as single biopsy sites [83, 102]. Reversion mutations have also been detected in circulating tumour DNA (ctDNA) in women who develop resistance to platinum [104-106] and in tumour tissue/ctDNA from patients with prostate and pancreatic cancer after progression following PARPi therapy [107-109]. Indeed, the use of temporal sampling of ctDNA may circumvent the challenges of determining differences in tumour heterogeneity and tumour evolution when analysing tumour tissue biopsies alone [110-112].

As yet, no prospective trial data are available regarding the incidence of reversion mutations in women with ovarian cancer who develop resistance to PARPi or their treatment responses to subsequent systemic anti-cancer therapy [70, 106]. Prospective trials are, therefore, required that utilise repeat tumour sampling and ctDNA to assess tumour evolution during therapy in women with $\mathrm{g} / \mathrm{s} B R C A \mathrm{mt}$ and $B R C A$ wt ovarian cancer. In this respect, serial molecular analyses of tumour evolution should parallel traditional methods of determining progressive disease such as clinical, biochemical and radiological assessment, especially in those women with mutations in genes that may initially confer sensitivity to PARPi therapy and then potentially revert/mutant to bring about resistance.

\section{Conclusion}

PARPi have anti-tumour activity as single agent therapy and offer effective maintenance treatment in recurrent, platinumsensitive high-grade serous ovarian carcinoma, with the greatest benefits being demonstrated in women with $\mathrm{g} / \mathrm{s} B R C A \mathrm{mt}$. Similar efficacy outcomes were demonstrated across a range of PARPi without any one drug showing superior efficacy. A number of class-based side effects have emerged, with early suggestions of particular drug-related side effects. Next generation sequencing-based assays that detect putative markers of HRD in tumour tissue or ctDNA, beyond, BRCA1/2 mutational status, may have potential as predictive biomarkers in certain clinical settings but further research is required.

Funding Dr. Robert D. Morgan is supported by a research grant from AstraZeneca and The Christie Hospital NHS Foundation Trust.

\section{Compliance with ethical standards}

Conflict of interest Dr. Robert D. Morgan is supported by a research grant from AstraZeneca and The Christie NHS Foundation Trust. Prof. Gordon C. Jayson and Dr. Andrew R. Clamp have received research grants from AstraZeneca. Prof. D. Gareth R. Evans has received travel grants from AstraZeneca and Amgen. Prof. Richard J Edmondson declares that he has no conflict of interest.

Human and animal statement This article does not contain any studies with human participants or animals performed by any of the authors.

Open Access This article is distributed under the terms of the Creative Commons Attribution 4.0 International License (http://creativeco mmons.org/licenses/by/4.0/), which permits unrestricted use, distribution, and reproduction in any medium, provided you give appropriate credit to the original author(s) and the source, provide a link to the Creative Commons license, and indicate if changes were made.

\section{References}

1. Jayson GC, Kohn EC, Kitchener HC, Ledermann JA (2014) Ovarian cancer Lancet 384:1376-1388

2. Ledermann JA (2017) Front-line therapy of advanced ovarian cancer: new approaches. Ann Oncol 28:viii46-viii50

3. Pujade-Lauraine E (2017) New treatments in ovarian cancer. Ann Oncol 28:viii57-viii60

4. Jayson GC, Kerbel R, Ellis LM, Harris AL (2016) Antiangiogenic therapy in oncology: current status and future directions. Lancet 388:518-529 
5. Ledermann JA (2016) PARP inhibitors in ovarian cancer. Ann Oncol 27(Suppl 1):i40-i44

6. Bryant HE, Schultz N, Thomas HD et al (2005) Specific killing of BRCA2-deficient tumours with inhibitors of poly(ADP-ribose) polymerase. Nature 434:913-917

7. Farmer H, McCabe N, Lord CJ et al (2005) Targeting the DNA repair defect in BRCA mutant cells as a therapeutic strategy. Nature 434:917-921

8. Heyer WD, Ehmsen KT, Liu J (2010) Regulation of homologous recombination in eukaryotes. Annu Rev Genet 44:113-139

9. Lilyquist J, LaDuca H, Polley E et al (2017) Frequency of mutations in a large series of clinically ascertained ovarian cancer cases tested on multi-gene panels compared to reference controls. Gynecol Oncol 147:375-380

10. Loveday C, Turnbull C, Ramsay E et al (2011) Germline mutations in RAD51D confer susceptibility to ovarian cancer. Nat Genet 43:879-882

11. Meindl A, Hellebrand H, Wiek C et al (2010) Germline mutations in breast and ovarian cancer pedigrees establish RAD51C as a human cancer susceptibility gene. Nat Genet 42:410-414

12. Norquist BM, Harrell MI, Brady MF et al (2016) Inherited mutations in women with ovarian carcinoma. JAMA Oncol 2:482-490

13. Rafnar T, Gudbjartsson DF, Sulem P et al (2011) Mutations in BRIP1 confer high risk of ovarian cancer. Nat Genet 43:1104-1107

14. Walsh T, Casadei S, Lee MK et al (2011) Mutations in 12 genes for inherited ovarian, fallopian tube, and peritoneal carcinoma identified by massively parallel sequencing. Proc Natl Acad Sci USA 108:18032-18037

15. Pelttari LM, Heikkinen T, Thompson D et al (2011) RAD51C is a susceptibility gene for ovarian cancer. Hum Mol Genet 20:3278-3288

16. Antoniou A, Pharoah PD, Narod S et al (2003) Average risks of breast and ovarian cancer associated with BRCA1 or BRCA2 mutations detected in case series unselected for family history: a combined analysis of 22 studies. Am J Hum Genet 72:1117-1130

17. Nielsen FC, van Overeem Hansen T, Sorensen CS (2016) Hereditary breast and ovarian cancer: new genes in confined pathways. Nat Rev Cancer 16:599-612

18. Bowtell DD (2010) The genesis and evolution of high-grade serous ovarian cancer. Nat Rev Cancer 10:803-808

19. Helleday T (2011) The underlying mechanism for the PARP and BRCA synthetic lethality: clearing up the misunderstandings. Mol Oncol 5:387-393

20. Tutt A, Bertwistle D, Valentine J et al (2001) Mutation in BRCA2 stimulates error-prone homology-directed repair of DNA doublestrand breaks occurring between repeated sequences. EMBO J 20:4704-4716

21. Venkitaraman AR (2014) Cancer suppression by the chromosome custodians, BRCA1 and BRCA2. Science 343:1470-1475

22. Venkitaraman AR (2009) Linking the cellular functions of BRCA genes to cancer pathogenesis and treatment. Annu Rev Pathol 4:461-487

23. Maxwell KN, Wubbenhorst B, Wenz BM et al (2017) BRCA locus-specific loss of heterozygosity in germline BRCA1 and BRCA2 carriers. Nat Commun 8:319

24. Riaz N, Blecua P, Lim RS et al (2017) Pan-cancer analysis of bi-allelic alterations in homologous recombination DNA repair genes. Nat Commun 8:857

25. Dougherty BA, Lai Z, Hodgson DR et al (2017) Biological and clinical evidence for somatic mutations in BRCA1 and BRCA2 as predictive markers for olaparib response in high-grade serous ovarian cancers in the maintenance setting. Oncotarget 8:43653-43661

26. Hennessy BT, Timms KM, Carey MS et al (2010) Somatic mutations in BRCA1 and BRCA2 could expand the number of patients that benefit from poly (ADP ribose) polymerase inhibitors in ovarian cancer. J Clin Oncol 28:3570-3576

27. Gibson BA, Kraus WL (2012) New insights into the molecular and cellular functions of poly(ADP-ribose) and PARPs. Nat Rev Mol Cell Biol 13:411-424

28. Rouleau M, Patel A, Hendzel MJ et al (2010) PARP inhibition: PARP1 and beyond. Nat Rev Cancer 10:293-301

29. Malanga M, Althaus FR (2005) The role of poly(ADP-ribose) in the DNA damage signaling network. Biochem Cell Biol 83:354-364

30. Murai J, Huang SY, Das BB et al (2012) Trapping of PARP1 and PARP2 by Clinical PARP Inhibitors. Cancer Res 72:5588-5599

31. Murai J, Huang SY, Renaud A et al (2014) Stereospecific PARP trapping by BMN 673 and comparison with olaparib and rucaparib. Mol Cancer Ther 13:433-443

32. Hopkins TA, Shi Y, Rodriguez LE et al (2015) Mechanistic dissection of PARP1 trapping and the impact on in vivo tolerability and efficacy of PARP inhibitors. Mol Cancer Res 13:1465-1477

33. McCormick A, Donoghue P, Dixon M et al (2017) Ovarian cancers harbor defects in nonhomologous end joining resulting in resistance to rucaparib. Clin Cancer Res 23:2050-2060

34. Patel AG, Sarkaria JN, Kaufmann SH (2011) Nonhomologous end joining drives poly(ADP-ribose) polymerase (PARP) inhibitor lethality in homologous recombination-deficient cells. Proc Natl Acad Sci USA 108:3406-3411

35. Ashworth A (2008) A synthetic lethal therapeutic approach: poly(ADP) ribose polymerase inhibitors for the treatment of cancers deficient in DNA double-strand break repair. J Clin Oncol 26:3785-3790

36. Kaelin WG Jr. (2005) The concept of synthetic lethality in the context of anticancer therapy. Nat Rev Cancer 5:689-698

37. Audeh MW, Carmichael J, Penson RT et al (2010) Oral poly(ADP-ribose) polymerase inhibitor olaparib in patients with BRCA1 or BRCA2 mutations and recurrent ovarian cancer: a proof-of-concept trial. Lancet 376:245-251

38. Coleman RL, Sill MW, Bell-McGuinn K et al (2015) A phase II evaluation of the potent, highly selective PARP inhibitor veliparib in the treatment of persistent or recurrent epithelial ovarian, fallopian tube, or primary peritoneal cancer in patients who carry a germline BRCA1 or BRCA2 mutation - An NRG Oncology/Gynecologic Oncology Group study. Gynecol Oncol 137:386-391

39. de Bono J, Ramanathan RK, Mina L et al (2017) Phase I, doseescalation, two-part trial of the PARP inhibitor talazoparib in patients with advanced germline BRCA1/2 mutations and selected sporadic cancers. Cancer Discov 7:620-629

40. Domchek SM, Aghajanian C, Shapira-Frommer R et al (2016) Efficacy and safety of olaparib monotherapy in germline BRCA1/2 mutation carriers with advanced ovarian cancer and three or more lines of prior therapy. Gynecol Oncol 140:199-203

41. Drew Y, Ledermann J, Hall G et al (2016) Phase 2 multicentre trial investigating intermittent and continuous dosing schedules of the poly(ADP-ribose) polymerase inhibitor rucaparib in germline BRCA mutation carriers with advanced ovarian and breast cancer. Br J Cancer 114:723-730

42. Fong PC, Boss DS, Yap TA et al (2009) Inhibition of poly(ADPribose) polymerase in tumors from BRCA mutation carriers. N Engl J Med 361:123-134

43. Fong PC, Yap TA, Boss DS et al (2010) Poly(ADP)-ribose polymerase inhibition: frequent durable responses in BRCA carrier ovarian cancer correlating with platinum-free interval. J Clin Oncol 28:2512-2519

44. Gelmon KA, Tischkowitz M, Mackay H et al (2011) Olaparib in patients with recurrent high-grade serous or poorly differentiated ovarian carcinoma or triple-negative breast cancer: a phase 
2, multicentre, open-label, non-randomised study. Lancet Oncol $12: 852-861$

45. Kaufman B, Shapira-Frommer R, Schmutzler RK et al (2015) Olaparib monotherapy in patients with advanced cancer and a germline BRCA1/2 mutation. J Clin Oncol 33:244-250

46. Kaye SB, Lubinski J, Matulonis U et al (2012) Phase II, openlabel, randomized, multicenter study comparing the efficacy and safety of olaparib, a poly (ADP-ribose) polymerase inhibitor, and pegylated liposomal doxorubicin in patients with BRCA1 or BRCA2 mutations and recurrent ovarian cancer. J Clin Oncol 30:372-379

47. Kristeleit R, Shapira-Frommer R, Oaknin A et al (2016) Clinical activity of the poly(ADP-ribose) polymerase (PARP) inhibitor rucaparib in patients (pts) with high-grade ovarian carcinoma (HGOC) and a BRCA mutation (BRCAmut): analysis of pooled data from study 10 (parts 1, 2a, and 3) and ARIEL2 (parts 1 and 2). Ann Oncol 27(suppl_6):8560

48. Kristeleit R, Shapiro GI, Burris HA et al (2017) A phase I-II study of the oral PARP inhibitor rucaparib in patients with germline BRCA1/2-mutated ovarian carcinoma or other solid tumors. Clin Cancer Res 23:4095-4106

49. Mateo J, Moreno V, Gupta A et al (2016) An adaptive study to determine the optimal dose of the tablet formulation of the PARP inhibitor olaparib. Target Oncol 11:401-415

50. Matulonis UA, Penson RT, Domchek SM et al (2016) Olaparib monotherapy in patients with advanced relapsed ovarian cancer and a germline BRCA1/2 mutation: a multistudy analysis of response rates and safety. Ann Oncol 27:1013-1019

51. Sandhu SK, Schelman WR, Wilding G et al (2013) The poly(ADP-ribose) polymerase inhibitor niraparib (MK4827) in BRCA mutation carriers and patients with sporadic cancer: a phase 1 dose-escalation trial. Lancet Oncol 14:882-892

52. Steffensen KD, Adimi P, Jakobsen A (2017) Veliparib monotherapy to patients with BRCA germ line mutation and platinum-resistant or partially platinum-sensitive relapse of epithelial ovarian cancer: a phase I/II study. Int J Gynecol Cancer 27:1842-1849

53. Swisher EM, Lin KK, Oza AM et al (2017) Rucaparib in relapsed, platinum-sensitive high-grade ovarian carcinoma (ARIEL2 Part 1): an international, multicentre, open-label, phase 2 trial. Lancet Oncol 18:75-87

54. Oza AM, Tinker AV, Oaknin A et al (2017) Antitumor activity and safety of the PARP inhibitor rucaparib in patients with highgrade ovarian carcinoma and a germline or somatic BRCA1 or BRCA2 mutation: Integrated analysis of data from Study 10 and ARIEL2. Gynecol Oncol

55. Lee JM, Cimino-Mathews A, Peer CJ et al (2017) Safety and clinical activity of the programmed death-ligand 1 inhibitor durvalumab in combination with poly (ADP-Ribose) polymerase inhibitor olaparib or vascular endothelial growth factor receptor 1-3 inhibitor cediranib in women's cancers: a dose-escalation, phase I study. J Clin Oncol 35:2193-2202

56. Liu JF, Barry WT, Birrer M et al (2014) Combination cediranib and olaparib versus olaparib alone for women with recurrent platinum-sensitive ovarian cancer: a randomised phase 2 study. Lancet Oncol 15:1207-1214

57. Matulonis UA, Wulf GM, Barry WT et al (2017) Phase I dose escalation study of the PI3kinase pathway inhibitor BKM120 and the oral poly (ADP ribose) polymerase (PARP) inhibitor olaparib for the treatment of high-grade serous ovarian and breast cancer. Ann Oncol 28:512-518

58. Oza A, Combe P, Ledermann J et al (2017) Evaluation of tumour responses and olaparib efficacy in platinum-sensitive relapsed ovarian cancer patients (PSROC) with or without measurable disease in the SOLO2 trial (ENGOT Ov-21). Ann Oncol 28:P344 (abstract 965P)
59. Coleman RL, Oza AM, Lorusso D et al. (2017) Rucaparib maintenance treatment for recurrent ovarian carcinoma after response to platinum therapy (ARIEL3): a randomised, double-blind, placebo-controlled, phase 3 trial. Lancet 390:1949-196

60. Ledermann J, Harter P, Gourley C et al (2012) Olaparib maintenance therapy in platinum-sensitive relapsed ovarian cancer. $\mathrm{N}$ Engl J Med 366:1382-1392

61. Ledermann J, Harter P, Gourley C et al (2014) Olaparib maintenance therapy in patients with platinum-sensitive relapsed serous ovarian cancer: a preplanned retrospective analysis of outcomes by BRCA status in a randomised phase 2 trial. Lancet Oncol 15:852-861

62. Mirza MR, Matulonis UA (2017) Niraparib in recurrent ovarian cancer. N Engl J Med 376:801-802

63. Pujade-Lauraine E, Ledermann JA, Selle F et al. (2017) Olaparib tablets as maintenance therapy in patients with platinum-sensitive, relapsed ovarian cancer and a BRCA1/2 mutation (SOLO2/ ENGOT-Ov21): a double-blind, randomised, placebo-controlled, phase 3 trial. Lancet Oncol 18:1274-1284

64. Ledermann JA, Harter P, Gourley C et al (2016) Overall survival in patients with platinum-sensitive recurrent serous ovarian cancer receiving olaparib maintenance monotherapy: an updated analysis from a randomised, placebo-controlled, double-blind, phase 2 trial. Lancet Oncol 17:1579-1589

65. Matulonis UA, Harter P, Gourley C et al (2016) Olaparib maintenance therapy in patients with platinum-sensitive, relapsed serous ovarian cancer and a BRCA mutation: Overall survival adjusted for postprogression poly(adenosine diphosphate ribose) polymerase inhibitor therapy. Cancer 122:1844-1852

66. Barber LJ, Sandhu S, Chen L et al (2013) Secondary mutations in BRCA2 associated with clinical resistance to a PARP inhibitor. J Pathol 229:422-429

67. Edwards SL, Brough R, Lord CJ et al (2008) Resistance to therapy caused by intragenic deletion in BRCA2. Nature 451:1111-1115

68. Sakai W, Swisher EM, Jacquemont C et al (2009) Functional restoration of BRCA2 protein by secondary BRCA2 mutations in BRCA2-mutated ovarian carcinoma. Cancer Res 69:6381-6386

69. Sakai W, Swisher EM, Karlan BY et al (2008) Secondary mutations as a mechanism of cisplatin resistance in BRCA2-mutated cancers. Nature 451:1116-1120

70. Ang JE, Gourley C, Powell CB et al (2013) Efficacy of chemotherapy in BRCA1/2 mutation carrier ovarian cancer in the setting of PARP inhibitor resistance: a multi-institutional study. Clin Cancer Res 19:5485-5493

71. Matulonis U, Herrstedt J, Tinker A et al (2017) Long-term benefits of niraparib treatment of recurrent ovarian cancer (OC). J Clin Oncol 35(suppl_15):5534

72. Fabbro M, Moore KN, Dorum A et al (2017) Safety and efficacy of niraparib in elderly patients with recurrent ovarian cancer. Ann Oncol 28:P332 (abstract 934PD).

73. Friedlander M, Gebski V, Gibbs E et al. (2017) Health-related quality of life (HRQOL) and patient-centred outcomes with maintenance olaparib compared with placebo following chemotherapy in patients with germline (g) BRCA-mutated (m) platinum-sensitive relapsed serous ovarian cancer (PSR SOC): SOLO2 phase 3 trial. J Clin Oncol 35(suppl_15):5507

74. Ledermann J, Lortholary A, Penson RT et al (2017) Adverse events (AEs) with maintenance olaparib tablets in patients (pts) with BRCA-mutated (BRCAm) platinum-sensitive relapsed serous ovarian cancer (PSR SOC): Phase III SOLO2 trial. J Clin Oncol 35(suppl_15):5518

75. Oza AM, Matulonis UA, Malander S et al (2017) Quality of life in patients with recurrent ovarian cancer (OC) treated with niraparib: results from ENGOT-OV16/NOVA trial. Ann Oncol 28(suppl_5):v330-v330 
76. Clamp A, Jayson G (2015) PARP inhibitors in BRCA mutationassociated ovarian cancer. Lancet Oncol 16:10-12

77. Oza AM, Cibula D, Benzaquen AO et al (2015) Olaparib combined with chemotherapy for recurrent platinum-sensitive ovarian cancer: a randomised phase 2 trial. Lancet Oncol 16:87-97

78. Gonzalez-Martin A, Backes FJ, Baumann KH et al (2016) A randomized, double-blind phase III trial niraparib maintenance treatment in patients with HRD + advanced ovarian cancer after response to front-line platinum-based chemotherapy. J Clin Oncol 34(suppl_15):TPS5606

79. Alsop K, Fereday S, Meldrum C et al (2012) BRCA mutation frequency and patterns of treatment response in BRCA mutationpositive women with ovarian cancer: a report from the Australian Ovarian Cancer Study Group. J Clin Oncol 30:2654-2663

80. Cancer Genome Atlas Research N. Integrated genomic analyses of ovarian carcinoma. Nature 2011; 474: 609-615

81. Cunningham JM, Cicek MS, Larson NB et al (2014) Clinical characteristics of ovarian cancer classified by BRCA1, BRCA2, and RAD51C status. Sci Rep 4:4026

82. Kanchi KL, Johnson KJ, Lu C et al (2014) Integrated analysis of germline and somatic variants in ovarian cancer. Nat Commun 5:3156

83. Patch AM, Christie EL, Etemadmoghadam D et al (2015) Wholegenome characterization of chemoresistant ovarian cancer. Nature 521:489-494

84. Pennington KP, Walsh T, Harrell MI et al (2014) Germline and somatic mutations in homologous recombination genes predict platinum response and survival in ovarian, fallopian tube, and peritoneal carcinomas. Clin Cancer Res 20:764-775

85. Gottipati P, Vischioni B, Schultz N et al (2010) Poly(ADP-ribose) polymerase is hyperactivated in homologous recombinationdefective cells. Cancer Res 70:5389-5398

86. McCabe N, Turner NC, Lord CJ et al (2006) Deficiency in the repair of DNA damage by homologous recombination and sensitivity to poly(ADP-ribose) polymerase inhibition. Cancer Res 66:8109-8115

87. Watkins JA, Irshad S, Grigoriadis A, Tutt AN (2014) Genomic scars as biomarkers of homologous recombination deficiency and drug response in breast and ovarian cancers. Breast Cancer Res 16:211

88. Ryland GL, Doyle MA, Goode D et al (2015) Loss of heterozygosity: what is it good for? BMC Med Genomics 8:45

89. Birkbak NJ, Wang ZC, Kim JY et al (2012) Telomeric allelic imbalance indicates defective DNA repair and sensitivity to DNA-damaging agents. Cancer Discov 2:366-375

90. Popova T, Manie E, Rieunier G et al (2012) Ploidy and large-scale genomic instability consistently identify basal-like breast carcinomas with BRCA1/2 inactivation. Cancer Res 72:5454-5462

91. Abkevich V, Timms KM, Hennessy BT et al (2012) Patterns of genomic loss of heterozygosity predict homologous recombination repair defects in epithelial ovarian cancer. Br J Cancer 107:1776-1782

92. Mukhopadhyay A, Elattar A, Cerbinskaite A et al (2010) Development of a functional assay for homologous recombination status in primary cultures of epithelial ovarian tumor and correlation with sensitivity to poly(ADP-ribose) polymerase inhibitors. Clin Cancer Res 16:2344-2351

93. Mukhopadhyay A, Plummer ER, Elattar A et al (2012) Clinicopathological features of homologous recombination-deficient epithelial ovarian cancers: sensitivity to PARP inhibitors, platinum, and survival. Cancer Res 72:5675-5682

94. Telli ML, Timms KM, Reid J et al (2016) Homologous recombination deficiency (HRD) score predicts response to platinumcontaining neoadjuvant chemotherapy in patients with triplenegative breast cancer. Clin Cancer Res 22:3764-3773

95. Wilcoxen KM, Becker M, Neff C et al (2015) Use of homologous recombination deficiency (HRD) score to enrich for niraparib sensitive high grade ovarian tumors. J Clin Oncol 33(suppl_15):5532

96. Frampton GM, Fichtenholtz A, Otto GA et al (2013) Development and validation of a clinical cancer genomic profiling test based on massively parallel DNA sequencing. Nat Biotechnol 31:1023-1031

97. Coleman RL, Swisher EM, Oza AM et al (2016) Refinement of prespecified cutoff for genomic loss of heterozygosity $(\mathrm{LOH})$ in ARIEL2 part 1: A phase II study of rucaparib in patients (pts) with high grade ovarian carcinoma (HGOC). J Clin Oncol 34(suppl_15):5540

98. O’Malley DM, Coleman RL, Oza A et al (2017) Results from the phase 3 study ARIEL3: mutations in non-BRCA homologous recombination repair genes confer sensitivity to maintenance treatment with the PARP inhibitor rucaparib in patients with recurrent platinum-sensitive high-grade ovarian carcinoma. In: AACR-NCI-EORTC molecular targets and cancer therapeutics conference

99. Lord CJ, Ashworth A (2013) Mechanisms of resistance to therapies targeting BRCA-mutant cancers. Nat Med 19:1381-1388

100. Norquist B, Wurz KA, Pennil CC et al (2011) Secondary somatic mutations restoring BRCA $1 / 2$ predict chemotherapy resistance in hereditary ovarian carcinomas. J Clin Oncol 29:3008-3015

101. Swisher EM, Sakai W, Karlan BY et al (2008) Secondary BRCA1 mutations in BRCA1-mutated ovarian carcinomas with platinum resistance. Cancer Res 68:2581-2586

102. Kondrashova O, Nguyen M, Shield-Artin K et al. (2017) Secondary somatic mutations restoring RAD51C and RAD51D associated with acquired resistance to the PARP inhibitor rucaparib in high-grade ovarian carcinoma. Cancer Discov 7:984-998

103. Lheureux S, Bruce JP, Burnier JV et al (2017) Somatic BRCA1/2 recovery as a resistance mechanism after exceptional response to poly (ADP-ribose) polymerase inhibition. J Clin Oncol 35:1240-1249

104. Christie EL, Fereday S, Doig K et al (2017) Reversion of BRCA1/2 germline mutations detected in circulating tumor DNA from patients with high-grade serous ovarian cancer. J Clin Oncol 35:1274-1280

105. Mayor P, Gay LM, Lele S, Elvin JA (2017) BRCA1 reversion mutation acquired after treatment identified by liquid biopsy. Gynecol Oncol Rep 21:57-60

106. Weigelt B, Comino-Mendez I, de Bruijn I et al. (2017) Diverse BRCA1 and BRCA2 reversion mutations in circulating cell-free DNA of therapy-resistant breast or ovarian cancer. Clin Cancer Res 23:6708-6720

107. Goodall J, Mateo J, Yuan W et al (2017) Circulating cell-free DNA to guide prostate cancer treatment with PARP inhibition. Cancer Discov 7:1006-1017

108. Quigley D, Alumkal JJ, Wyatt AW et al (2017) Analysis of circulating cell-free DNA identifies multiclonal heterogeneity of BRCA2 reversion mutations associated with resistance to PARP inhibitors. Cancer Discov 7:999-1005

109. Pishvaian MJ, Biankin AV, Bailey P et al (2017) BRCA2 secondary mutation-mediated resistance to platinum and PARP inhibitor-based therapy in pancreatic cancer. Br J Cancer 116:1021-1026

110. Wan JC, Massie C, Garcia-Corbacho J et al (2017) Liquid biopsies come of age: towards implementation of circulating tumour DNA. Nat Rev Cancer 17:223-238

111. Parkinson CA, Gale D, Piskorz AM et al (2016) Exploratory analysis of TP53 mutations in circulating tumour DNA as biomarkers of treatment response for patients with relapsed high-grade serous ovarian carcinoma: a retrospective study. PLoS Med 13:e1002198

112. Murtaza M, Dawson SJ, Tsui DW et al (2013) Non-invasive analysis of acquired resistance to cancer therapy by sequencing of plasma DNA. Nature 497:108-112 